\title{
Types of Psychiatric Presentation to Emergency Room of a Tertiary Care Center: A Record-based Retrospective Study
}

\author{
Mohmmed Kamran Chisty ${ }^{1}$, Ganesh Kini ${ }^{2}$, Anil Kakunje ${ }^{3}$, Ravichandra Karkal ${ }^{4}$, Perla Mohammed Ali Nishad ${ }^{5}$
}

\begin{abstract}
Background: Emergency psychiatry is a developing subspecialty, and emergency rooms (ERs) are the major entry point for people with mental illness. Emergency room psychiatrists work under circumstances of uncertainty and time limitations. The knowledge of various ER psychiatric presentations helps us to make tailor-made intervention.

Aim and objective: The aim of the study was to examine the various clinical presentations of psychiatric patients brought to the ER.

Materials and methods: It is a register-based study, and patients who attended the ER of Yenepoya Medical College Hospital (YMCH) constituted the study sample.

Results: A total of 134 patients attended the ER of YMCH during the study period. The highest number of patients who were bought to the emergency was of bipolar affective disorder (BPAD) followed by patients with depression, and the least presentation was for obsessive-compulsive disorder (OCD) and dissociative disorders.

Conclusions: The nature of the emergency with which patients sought emergency services in India is varied.

Keywords: Emergency psychiatry, Emergency room presentation, Register-based study.

Indian Journal of Private Psychiatry (2020): 10.5005/jp-journals-10067-0051
\end{abstract}

\section{INTRODUCTION}

Emergency rooms (ERs) are a major point of entry to acute psychiatric services for persons with severe mental illness, particularly for those who are violent. Emergency psychiatry has developed as a medical subspecialty in the last few decades. The goal of psychiatric emergency services is to provide treatment at the least restrictive level of care for patients with an acute psychiatric problem. ' Usually, adults present with multiple problems in the ER. Substance-use disorders, suicide attempts, and self-destructive behavior are the usual problems with which adolescents present to ER. They account for $20 \%$ of the psychiatric ER population. Geriatric patients account for $2-5 \%$ of psychiatric ER presentations. ${ }^{2}$ Certain groups of patients like rape victims and victims of domestic violence require emergency medical and forensic services in addition to psychiatric services. Emergency rooms are a major point of entry to acute psychiatric services for persons with severe mental illness, particularly for those who are violent. An emergency psychiatrist is called to treat patients who are prone to violence and sometimes to predict the likelihood of future violence. ${ }^{3}$ On the other hand, psychiatric patients have been found to exhibit particular risk factors for homelessness and experience less adequate support from family and friends. ${ }^{4}$ Homeless patients present with more negative symptoms and higher rates of psychosis than their domiciled counterparts. ${ }^{5}$ Emergency rooms psychiatrists work under circumstances of considerable uncertainty like limitations of time, unclear history, and decisions that have to be made with limited information. Usually, register-based studies in psychiatry are conducted on inpatients. With deinstitutionalization, psychiatric patients are increasingly being treated in outpatient rooms and ER. ${ }^{6}$

This study attempts to examine the various clinical presentations of psychiatric patients brought to the ER. We hope this study helps us to develop a tailor-made intervention, which can be delivered at the ER. To the best of our knowledge, no study in India has been done on presentation.
${ }^{1-5}$ Department of Psychiatry, Yenepoya Medical College, Mangaluru, Karnataka, India

Corresponding Author: Mohmmed K Chisty, Department of Psychiatry, Yenepoya Medical College, Mangaluru, Karnataka, India, Phone: +91 9902233743, e-mail: kamranchisty@gmail.com

How to cite this article: Chisty MK, Kini G, Kakunje A, et al. Types of Psychiatric Presentation to Emergency Room of a Tertiary Care Center: A Record-based Retrospective Study. Ind J Priv Psychiatry 2020;14(1): 6-8.

Source of support: Nil

Conflict of interest: None

\section{Materials and Methods}

Patients who attended the ER of YMCH from July 2018 to December 2018 for the psychiatric problem constituted the study population. Details of all psychiatry patients who presented to ER during the study period and patients who were referred from the other departments were also included in the study. The study was conducted after obtaining ethical clearance from the Department of Ethics, Yenepoya Medical College bearing protocol no-YEC$1 / 2019 / 185$. Clinical and sociodemographic details of the patients obtained using a register maintained by the medical record department of $\mathrm{YMCH}$. The data were categorized according to the International Classification of Diseases version 10. The data were tabulated in a Microsoft Excel spreadsheet under appropriate columns. Pivot charts were created in Microsoft Excel, and the data were grouped accordingly.

\section{RESULTS}

A total of 134 patients attended the ER of YMCH during the 6 months period. Among them, 116 were males (rural $=70$, urban $=46$ ) and 
66 were females (rural $=7$, urban $=59$ ). The most frequent diagnosis for which ER psychiatric treatment was sought was bipolar affective disorder (BPAD) $(n=41)$, among whom 16 were male with a rural background with a mean age of $31.53 \pm 8.36,13$ were male patients with an urban background with a mean age of $31.53 \pm 8.36$, and 3 were female from an urban background with a mean age of $25.33 \pm 2.30$ as shown in Table 1. The patients with depression $(n=36)$ have secondhighest presentation; most of the patients presented with worsening of somatoform/insomnia. Nineteen male patients with depression with a mean age of $42.73 \pm 12.56$ were from a rural background and 13 males with a mean age of $41.61 \pm 11.04$ were from an urban background. Thirty-two patients came for alcohol dependence syndrome (ADS) and associated complications with either withdrawal seizures or delirium. None of the patients sought ER for deaddiction. Schizophrenia patients $(n=20)$ are presented to ER with various manifestations, and most of the patients with schizophrenia were bought to YMCH by social workers due to exaggerations of symptoms mostly due to poor adherence to treatment. Patients with panic disorder ( $n=12$ ) usually presented to the ER at midnight. In the 6 months of study, only one patient for dissociative disorder and one patient for obsessive-compulsive disorder (OCD) presented to ER as shown in Table 2, and the same is depicted in Figure 1.

\section{Discussion}

In our study, we found that $30 \%$ of the patients who sought treatment in the ER were having a diagnosis of BPAD. This result is comparable to a study conducted in AlIMS Delhi by Kumar et al. ${ }^{7}$ Patients with BPAD during manic episodes are extremely violent and are easily recognized by family members and they come to medical attention immediately. Irritability and violence by patients are frequently referred to tertiary care centers like $\mathrm{YMCH}$ as the medical officers, nursing staff, resident doctors, and ancillary workers in primary health care centers are not sufficiently trained on how to handle violence by patients with mental health conditions. In our study, most of the patients had a diagnosis of severe depression with/without psychotic symptoms, and the patient presented with either worsening of somatic symptoms or insomnia. In the study done by Jain et al. a significant number of patients who presented to the ER with depression had attempted suicide. ${ }^{8}$ The disparity between our result and the study conducted by Jain et al. could be due to the fact that the primary catchment area for YMCH is the Kasargod district, Kerala, which is a Muslim majority district. Since suicide is forbidden in Islam, even though patients have suicidal thoughts they had not attempted suicide. Alcohol dependence syndrome was the next commonest presentation in our study as $17 \%$ of the samples were diagnosed to have ADS, which is comparable to the previous study conducted by Richoux et al. in Cochin. ${ }^{9}$ Most of our patients with schizophrenia had an exacerbation of psychotic symptoms due to poor adherence and were bought by social workers. Our schizophrenia patients are less frequently presented with concurrent substance use and violence than the Western countries where there is easy access to guns. In the study done by Dani and Thienhaus in Washington DC, they

Table 1: Mean and standard deviations (SD) of age, gender, and regional distribution of patients presented to the emergency room

\begin{tabular}{|c|c|c|c|c|}
\hline \multirow[b]{2}{*}{ Diagnosis } & \multicolumn{2}{|c|}{ Rural } & \multicolumn{2}{|c|}{ Urban } \\
\hline & Male & Female & Male & Female \\
\hline Alcohol dependence syndrome & $42.12 \pm 9.20$ & NA & $32.25 \pm 9.25$ & $39.5 \pm 9.19$ \\
\hline Bipolar affective disorder & $31.53 \pm 8.36$ & NA & $31.53 \pm 8.36$ & $25.33 \pm 2.30$ \\
\hline Depression & $42.73 \pm 12.56$ & NA & $41.61 \pm 11.04$ & $45.33 \pm 12.50$ \\
\hline Dissociative motor disorder & - & - & NA & - \\
\hline Osessive-compulsive disorder & - & - & NA & - \\
\hline Panic disorder & - & NA & $29 \pm 11.28$ & $28.5 \pm 5.06$ \\
\hline Schizophrenia & $35.75 \pm 9.71$ & NA & $36 \pm 9.48$ & NA \\
\hline
\end{tabular}

Note: NA, mean and SD not available due to a single sample; -, no data available

Table 2: The age, gender, and regional distribution of patients presented to the emergency room are shown here

\begin{tabular}{|c|c|c|c|c|c|c|c|}
\hline \multirow[b]{2}{*}{ Diagnosis } & \multicolumn{2}{|c|}{ Rural } & \multirow[b]{2}{*}{ Rural total } & \multicolumn{2}{|c|}{ Urban } & \multirow[b]{2}{*}{ Urban total } & \multirow[b]{2}{*}{ Grand total } \\
\hline & Male & Female & & Male & Female & & \\
\hline Alcohol dependence syndrome & 16 & 1 & 17 & 4 & 2 & 6 & 23 \\
\hline Bipolar affective disorder & 23 & 2 & 25 & 13 & 3 & 16 & 41 \\
\hline Depression & 19 & 1 & 20 & 13 & 3 & 16 & 36 \\
\hline Dissociative motor disorder & & & & 1 & & 1 & 1 \\
\hline Osessive-compulsive disorder & & & & 1 & & 1 & 1 \\
\hline Panic disorder & & 1 & 1 & 7 & 4 & 11 & 12 \\
\hline Schizophrenia & 12 & 1 & 13 & 6 & 1 & 7 & 20 \\
\hline Grand total & 70 & 7 & 77 & 46 & 13 & 59 & 134 \\
\hline
\end{tabular}




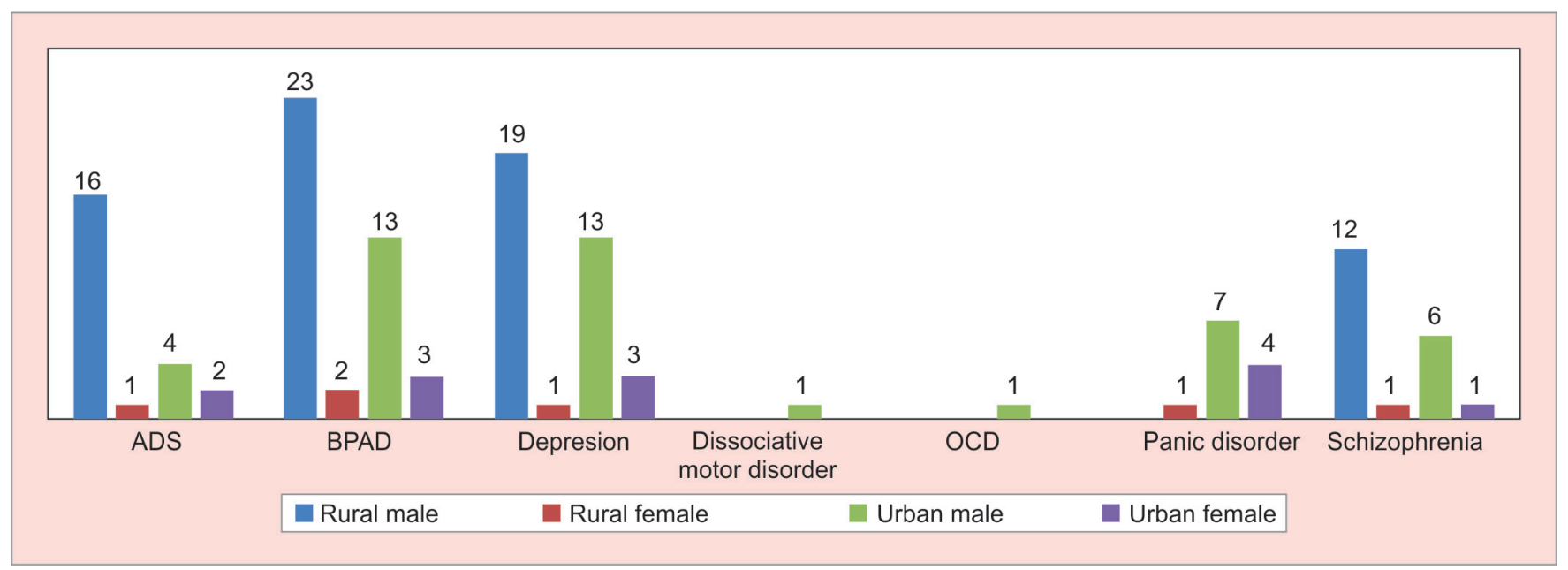

Fig. 1: Distribution of gender to the emergency presentations to the emergency room. ADS, alcohol dependence syndrome; BPAD, bipolar affective disorder; OCD, osessive-compulsive disorder

showed that patients in the USA who have been diagnosed with schizophrenia presented with severe complaints and are more functionally impaired. ${ }^{10}$ The reason for less functional impairment in Indian schizophrenic patients might be that there is good family support, which leads to early treatment, and this leads to reduced duration of untreated psychosis. Panic disorder patients presented to the internal medicine department with symptoms similar to a respiratory or cardiac disorder later refer to the psychiatry department for evaluation. Pollard and Lewis reported that any misdiagnosis could have devastating long-term clinical and financial consequences. $^{11}$

In our study, no structured diagnostic interview nor severity rating scale was used for diagnosis or to understand the severity of the illness. The urgency of presentations could have been underestimated or overestimated by the investigator. Since many different clinicians had made diagnoses, inter-rater reliability in the current study could be one of the limitations. The small sample size may also be another limitation of the study, and the results of this study cannot be generalized to the entire population.

\section{Conclusion}

The nature of the emergency with which patients sought emergency service in India has variations and more such studies with a large sample size can help to formulate better treatment plans.

\section{References}

1. Schuckit AM. Alcohol-related disorders. In: Sadock BJ, Sadock VA, Ruiz P, editors. Kaplan and Sadock's Comprehensive Textbook of Psychiatry, 9th ed. (Vol. 1). Philadelphia: Lippincott Williams \& Wilkins; 2009.
2. Ellison JM, Hughes DH, White KA. An emergency psychiatry update. Hosp Community Psychiatry 1989 Mar;40(3):250-260. DOI: 10.1176/ ps.40.3.250.

3. Gerson S, Bassuk E. Psychiatric emergencies: an overview. Am J Psychiatry 1980;137(1):1-11. DOI: 10.1176/ajp.137.1.1.

4. Caton $C L$, Shrout $P E$, Eagle PF, et al. Risk factors for homelessness among schizophrenic men: a case-control study. Am J Public Health 1994 Feb;84(2):265-270. DOI: 10.2105/ajph.84.2.265.

5. Wu T, Serper MR. Social support and psychopathology in homeless patients presenting for emergency psychiatric treatment. J Clin Psychol 1999 Sep 1;55(9):1127-1133. DOI: 10.1002/(sici)10974679(199909)55:9<1127::aid-jclp9>3.0.co;2-m.

6. Skodol AE, Karasu TB. Emergency psychiatry and the assaultive patient. Am J Psychiatry 1978;135(2):202-205. DOI: 10.1176/ ajp.135.2.202.

7. Aggression and Psychosis in Patients Seeking Emergency Psychiatric Care in New Delhi, India | OMICS International [Internet]. [cited 2019 Dec 15]. Available from: https://www.omicsonline.org/ open-access/aggression-and-psychosis-in-patients-seekingemergency-psychiatric-care-in-new-delhi-india-1522-4821-1000251. php?aid $=61158$.

8. Jain V, Singh H, Gupta SC, et al. A study of hopelessness, suicidal intent and depression in cases of attempted suicide. Indian J Psychiatry 1999;41(2):122-130.

9. Richoux C, Ferrand I, Casalino E, et al. Alcohol use disorders in the emergency ward: choice of the best mode of assessment and identification of at-risk situations. Int J Emerg Med 2011 Jun 14;4(1):27. DOI: 10.1186/1865-1380-4-27.

10. Dani MM, Thienhaus OJ. Characteristics of patients with schizophrenia in two cities in the U.S. and India. Psychiatr Serv 1996 Mar;47(3):300301. DOI: 10.1176/ps.47.3.300.

11. Pollard CA, Lewis LM. Managing panic attacks in emergency patients. J Emerg Med 1989 Oct;7(5):547-552. DOI: 10.1016/07364679(89)90164-9. 